\title{
SECOND DISCUSSION SESSION
}

(Thursday morning; 7 September, 1972)

\section{Chairman: F. B. Wood}

F. B. Wood: Yesterday we had talks about light curves of eclipsing stars, and people drew on the blackboard to illustrate. Today I have a copy of an Atlas of Light Curves of Eclipsing Variables compiled by M. G. Fracastoro. I can't very well pass it around, but if you wish to look at it during coffee break or afterwards, you will see that we have a beautiful series of various light curves; if any of them illustrate your particular paper, I'm sure you'd be free to borrow it. I think that when Fracastoro prepared this, he compared it with catalogues that gave solutions for $L_{1}$ and $R_{1}$ and so forth by saying that while you can read the vital statistics of bathing beauties (36-28-34 or whatever) it isn't quite the same thing as looking at pictures of the girls themselves. Now we have the 'pictures'. I have tried to arrange the first few talks to fit in with yesterday's discussion. First, will Dr. Catalano talk on photometric evidence of gaseous envelopes in close binaries?

Catalano: I should like to make a few remarks on what we can infer about gaseous envelopes in close binaries from the analysis of absorption effects in the light curve at phases just preceding and following the primary minimum. Such absorption effects are shown in Figure 1, where the $V$ light curves around the primary minimum, of the systems V 548 Cyg (Rodonò, 1976), AR Lac (Blanco and Catalano, 1972), $\delta$ Lib (Koch, 1962) are given. Other systems could be added to these, such as RS Sgr (Baglow, 1948) and R CMa (Koch, 1960); U Oph (Huffer and Kopal, 1951) probably shows the same effect at the secondary minimum.

It is known that many systems show absorption effects in their light curves and atmospheric eclipses have been suggested in many cases, however what is interesting in the systems quoted is the duration and the depth of the disturbance.

The observed light curves could be explained assuming that the primary component is eclipsed by an extended semi-transparent envelope surrounding the secondary component. An estimate of the size of such envelopes can be made from the geometrical elements given in the literature and from the phases $\bar{\phi}_{\mathrm{abs}}$ in the light curves of the beginning and the end of the absorption features. These data, together with the radii obtained for the envelopes, measured in units of the separation of the two components, are given in Table I. The fractional radii are also given. We note that the envelope is nearly in contact with the surface of the primary component $\left(\varrho+r_{1} \approx 1, \varrho\right.$ fractional radius of the envelope and $r_{1}$ radius of the primary component). The values of the radii of the envelopes range from 0.6 for V $548 \mathrm{Cyg}$ to 0.8 for AR Lac and are about twice the stellar radii, and 2.5 in the case of AR Lac. These figures are very close to the ones Dr. Batten quoted in his review for the size of the disk observed around the primary components of Algol-type systems. It is clear that these results 


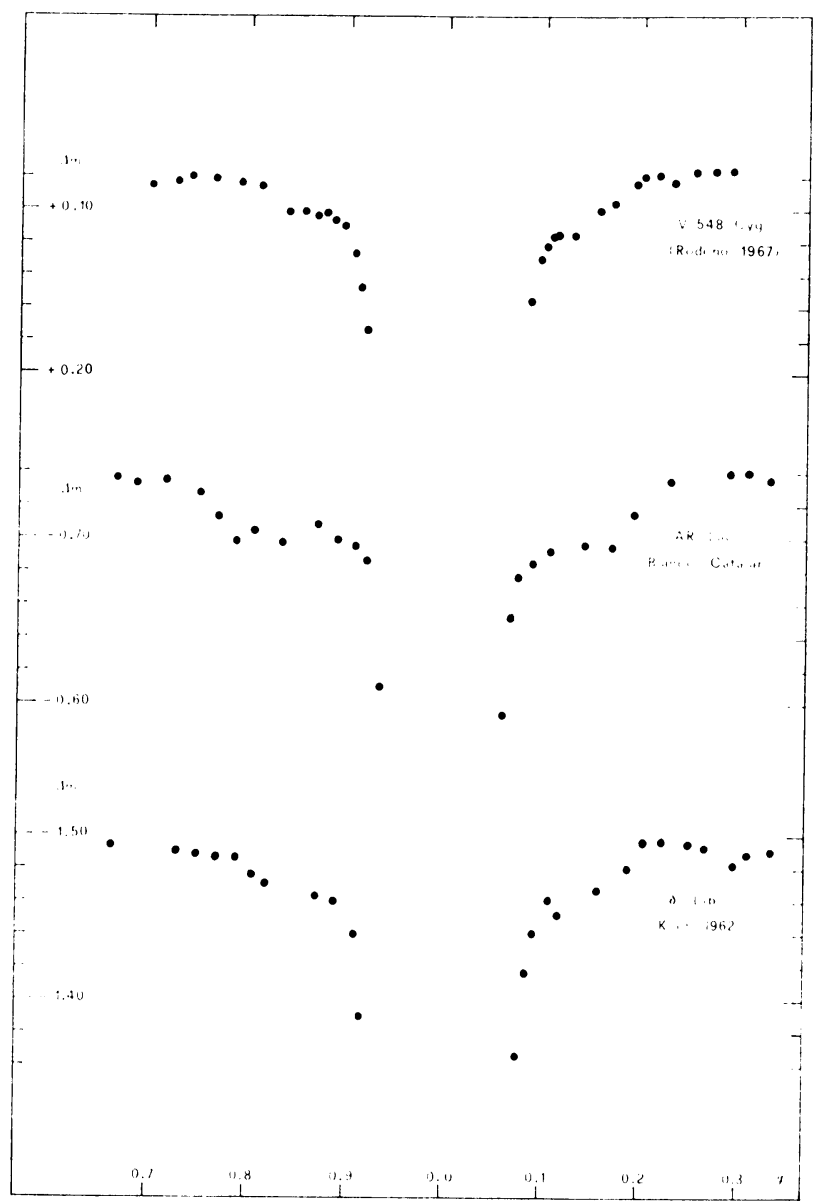

Fig. 1. Light curves of three eclipsing binary systems showing effects due to circumstellar matter.

TABLE I

Data for envelopes in five systems

\begin{tabular}{llllllll}
\hline System & Sp & $i$ & $r_{1}$ & $r_{2}$ & $\bar{\phi}_{\text {abs }}$ & $Q_{\text {env }}$ & $Q-r_{2}$ \\
& & & & & & & \\
R CMa & A9 + K5 & $77^{\circ}$ & 0.302 & 0.248 & $0^{P} .18$ & 0.61 & 0.36 \\
V 548 Cyg & A0 + F7 & 83 & 0.347 & 0.260 & $0^{P} .20$ & 0.60 & 0.34 \\
AR Lac & G2 + K0 & 90 & 0.194 & 0.319 & $0^{P} .25$ & 0.80 & 0.49 \\
$\delta$ Lib & A0 + G8 & 79 & 0.300 & 0.308 & $0^{P} .22$ & 0.68 & 0.37 \\
RS Sgr & B5 $:$ A3 & 82 & 0.326 & 0.261 & $0^{P} .21$ & 0.64 & 0.38
\end{tabular}


could be improved if a solution of the geometrical and light elements is carried on taking into account the atmospheric eclipse.

Considering now the plateau of the light curve during the absorption phases, we might think that the primary star is wholly eclipsed by the envelope for some time. However, if we compare the diameter of the eclipsed star, $2 r_{1}$, and the thickness, $\varrho-r_{2}$, of the envelope, we find that this can happen only for AR Lac. To overcome this difficulty without changing drastically the radii of the two components, we could describe the absorption effect by a tongue of matter streaming near the primary component as in Fletcher's (1964) model of Algol. Then, assuming that (i) the absorption is due to electron scattering, (ii) at least one tenth of the stellar disk is obscured, and (iii) the stream extends no more than the separation of the star surfaces, we find values of the density of the stream of $1.6 \times 10^{12}$ to $3.7 \times 10^{12}$ (Table II). These values agree fairly well with those found in other systems at least as far as the stream hypothesis is concerned. For AR Lac the envelope assumption leads to a mean density of about $10^{11}$ electron $\mathrm{cm}^{-3}$ (Table II).

\section{TABLE II}

Electron densities

$\begin{array}{lll}\text { System } & \begin{array}{l}N_{e} \mathrm{~cm}^{-3} \\ \text { (envelope) }\end{array} & \begin{array}{l}N_{e} \mathrm{~cm}^{-3} \\ \text { (stream) }\end{array} \\ \text { R CMa } & & 2.1 \times 10^{12} \\ \text { V 548 Cyg } & 1.0 \times 10^{11} & 2.8 \times 10^{12} \\ \text { AR Lac } & 3.1 \times 10^{12} \\ \delta \text { Lib } & & 1.6 \times 10^{12} \\ \text { RS Sgr } & & 3.0 \times 10^{12}\end{array}$

Oliver: I would like to comment on AR Lac which is apparently different from the other systems. The mass ratio of AR Lac is close to unity, which makes it a different kind of beast from the others. Kron (1947) observed quite substantial dips in the light curve (at one epoch, we can see three or four). If two of these just happened to be placed symmetrically on either side of primary minimum, they would produce the effect you are talking about. You estimate the envelope around the secondary star in AR Lac to extend to 0.8 of the separation, and that is well beyond the Roche limiting radius of 0.38 . This means the envelope does not belong to the secondary : it belongs to the system. Finally, AR Lac is one of the R CVn systems. The secondary component is a late-type $(\sim \mathrm{KO})$ subgiant and shows $\mathrm{H}$ and $\mathrm{K}$ emission lines in its spectrum. There are lots of photometric anomalies in systems of this sort that will probably be discussed later. I wonder if this system really belongs with the other four.

Catalano: The extension of the envelope is a problem, and also it is strange that we find an envelope around the secondary component. An alternative hypothesis is to suppose that there is a bright envelope around the primary component, but then there 
would be a problem about the density needed in the envelope to explain the loss of light we observed.

Oliver: Kron (1947) explained this phenomenon in the light curve of AR Lac by dark spots on the primary component.

Catalano: Yes, but I found that the light curve I obtained was rather different from Kron's. We showed some years ago that these variations in the light curve could be explained by variations in the light of the comparison star that Kron used. We have a stable light curve now, and the feature I showed repeats from cycle to cycle.

Oliver: Over how many cycles?

Catalano: We have observed it throughout a year, but Kron found that the spotted region on the primary star changed in about a month or less; and that is just the period we have found for the variations of the comparison star.

Oliver: I realize that, but, unfortunately, WY Cnc, SS Cam, and probably several others that are in other ways similar to AR Lac behave in the same way. For these stars it is certainly not variations in the comparison star that are responsible.

Van't Veer: Some of the systems in Table I do not seem to be quite possible dynamically. At least two or three systems are contact systems, or nearly so, as far as I can see from the radii. The width of the envelope $\left(\varrho-r_{2}\right)$ as quoted by Catalano, is a real problem in these systems. The envelope extends far beyond the Lagrangian points into regions where the forces are directed outward from the system. It is not clear to me how you can explain the shoulders of the light curve if there is a homogeneous envelope around the whole system.

Catalano: Yes, this is true. I did not set out to explain everything in this one report. I am just exploring the consequences of assuming a gaseous envelope around the secondary component.

Smak: Is there any spectroscopic evidence supporting your interpretation?

Catalano: Perhaps, in R CMa, of which Dr. Kitamura could tell us. Sanford (1951) also remarked of the spectrum of AR Lac: "flat, shallow profiles of the rapidly rotating secondary characterize the intervals... preceding and following primary minimum". Struve found that the $\mathrm{Ca}$ II emission lines originate in all parts of the stellar surface, and not in a limited region like a spot.

Hall: Is your definition of $\varrho$ the same as Dr. Batten's? Did he not find $\varrho$ to be approximately 0.3 ?

Batten: Yes, but I was talking about disks around primary components.

Hall: Dr. Catalano mentioned that I found a similar effect in the light curve of RS Cep. Just before first contact, and just after fourth contact, there is loss of light. You have found light losses of about 0.05 , but in the light curve of RS Cep it is a little greater. Two other Algol-type systems, AQ Peg and VW Cyg have been investigated by Bob Tate, as part of his Master's thesis, and they show light losses of a few hundredths of a magnitude. My interpretation (Hall, 1972) is quite different from yours. I put the envelope or disk around the primary star. These systems are known to display the type of emission ascribed to a ring in RW Tau, and I think of my disk as the relatively dense, inner portion of the ring, sufficiently bright even when seen 
through broad-band filters like $B$ and $V$ to have some surface brightness of their own. So I think of the dips in the light curve as the eclipse of that luminous disk by the cool star. Then for RS Cep, I obtain $\varrho \approx 0.35$ - in good agreement with average value found by Batten. The eclipses of AQ Peg and VW Cyg must be total, but the light curves are not exactly flat during totality - the light curves look more like those of annular eclipses. My interpretation is that the surface brightness of the disk is concentrated towards the limb of the hotter star. The cooler star does totally eclipse the hot star itself, but it does not totally eclipse the disk. The eclipse of the luminous disk is 'annular'. If the disk is around the hot star, it is within the Roche lobe, whereas Dr. Catalano's envelopes are outside the Roche lobe of the cooler star.

Fracastoro: I support Dr. Oliver's view on AR Lac; it looks to me, too, to be quite different from the other four systems. The spectrum of the primary star and the orbital inclination are different. Most of all, the value of $r_{1}$ is much smaller than in the other systems. I have another comment on what Dr. Hall says. I would be careful before speaking about dark clouds or bright clouds. Any solar observer can tell you that prominences look bright against the background of the sky, but dark against the surface of the Sun.

Hall: The loss of light just before first contact involves that part of the envelope or disk which is projected against the sky, not against the star.

Fracastoro: It would add light - but we observe a depression in the light curve.

Hall: It has some light of its own, and when the cool star moves in front of the envelope, you have an eclipse of the envelope.

Catalano: But we know systems with bright rings around the primary components - U Cep and RW Tau - which do not show this feature in their light curves. I don't know if these rings are denser and their light greater.

Hall: Oh no. In systems like RS Cep, the relative radius of the primary is quite small. In Dr. Huang's original way of visualizing the problem, there is more empty space in which to put a ring and build up quite a dense disk. In U Cep there is relatively little empty space, so it is harder to build up a substantial disk.

F. B. Wood: Let's conclude that AR Lac is a subject for a symposium on its own, and move along. Dr. Catalano wishes to present another topic, and then Dr. Hutchings has a presentation on spectroscopic aspects. We'll discuss the two together.

Catalano: Dr. Fracastoro and I have compared the frequencies of elliptical orbits among all spectroscopic binaries, that are also eclipsing binaries as obtained from Batten's catalogue (1968). Only $19 \%$ of the observed spectroscopic eccentricities are confirmed by photometric observations. Of 39 systems that show spectroscopic eccentricities that are not confirmed by the light curves, 17 show asymmetric light curves - the maxima are of different heights. There is a correlation between the sense of the asymmetry of these light curves and the spectroscopically derived value of the longitude of periastron, $\omega$. Systems for which $0^{\circ} \leqslant \omega \leqslant 180^{\circ}$ are fainter at the maximum following the primary minimum, and systems for which $180^{\circ} \leqslant \omega \leqslant 360^{\circ}$ are fainter at the maximum preceding the primary minimum.

Hutchings: The main part of what I want to say does not particularly tie in with 
the foregoing discussion. However, I would like to report on some computations which will bridge the gap as they apply to photometric observations of hot gas streams or envelopes in general. Figure 2 shows the effects on a light curve of an electron-scattering envelope centred on the hot component of a detached binary system. The density $\left(\sim 10^{12}\right.$ particles $\left.\mathrm{cm}^{-3}\right)$ and temperature $\left(\sim 10^{4} \mathrm{~K}\right)$ are of the order of these we have been discussing, and I have considered two geometrical configurations: an equatorial disk as drawn and a full sphere of the same outer radius. The effect is shown for two values of $i\left(90^{\circ}\right.$ for total eclipse and $72^{\circ}$ for a partial) and represents the deviation from the light-curve derived from the system without the envelope. Reasonable changes to the geometrical and other parameters changes absolute values by factors of up to $\sim 4$ but the general results are as illustrated. The main features are the drop in the light curve before first contact (and through it, in
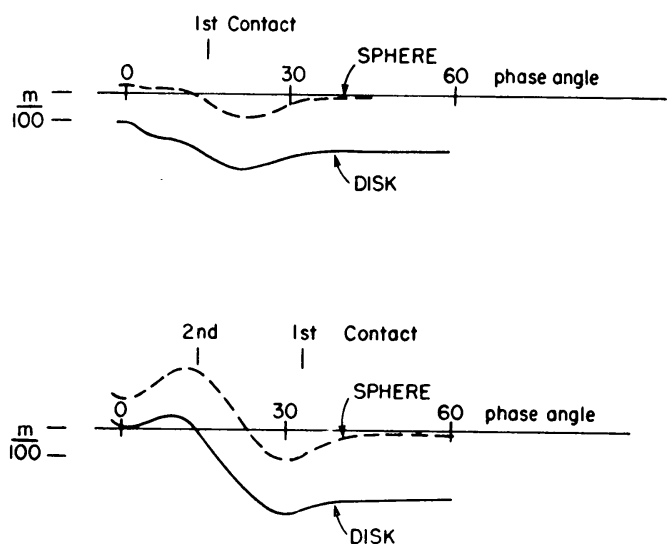

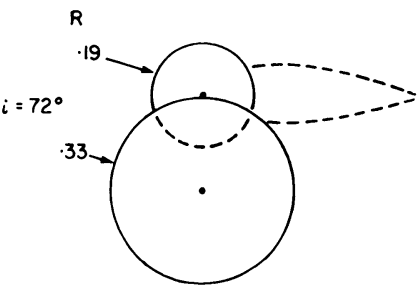

$i=90^{\circ}$

Fig. 2. Effect of electron-scattering disk on light curve of an eclipsing system around primary eclipse. The disk is assumed to extend around the primary star to three times its radius to have a density of $10^{12}$ electrons $\mathrm{cm}^{-3}$. and a temperature of about $10^{4} \mathrm{~K}$.

the total-eclipse case) of some one or two hundredths of a magnitude. Also, if the envelope is large enough, the eclipse depth is diminished. I would suggest, therefore, that this is an effect which may explain some of the pre-eclipse dips we have been looking at. I shall return to its relevance to my observations in a few minutes.

I should now like briefly to describe observations, both spectroscopic and photometric, of two emission-line stars which I think may be binary systems in the process of mass-exchange. They both have some extraordinary properties and I don't feel I understand them at this stage, so would welcome suggestions and discussion. The stars are HD 187399 (B7e) and 173219 (B0e). They are both shell stars of a sort and show dramatic, periodic spectrum variations.

In the Balmer and Ca II K lines in the spectrum of HD 187399 there are some points to notice: (i) the double absorption with variable separation; (ii) the strong increase in the blue-shifted absorption, accompanied by P Cyg emission at the periastron. I 


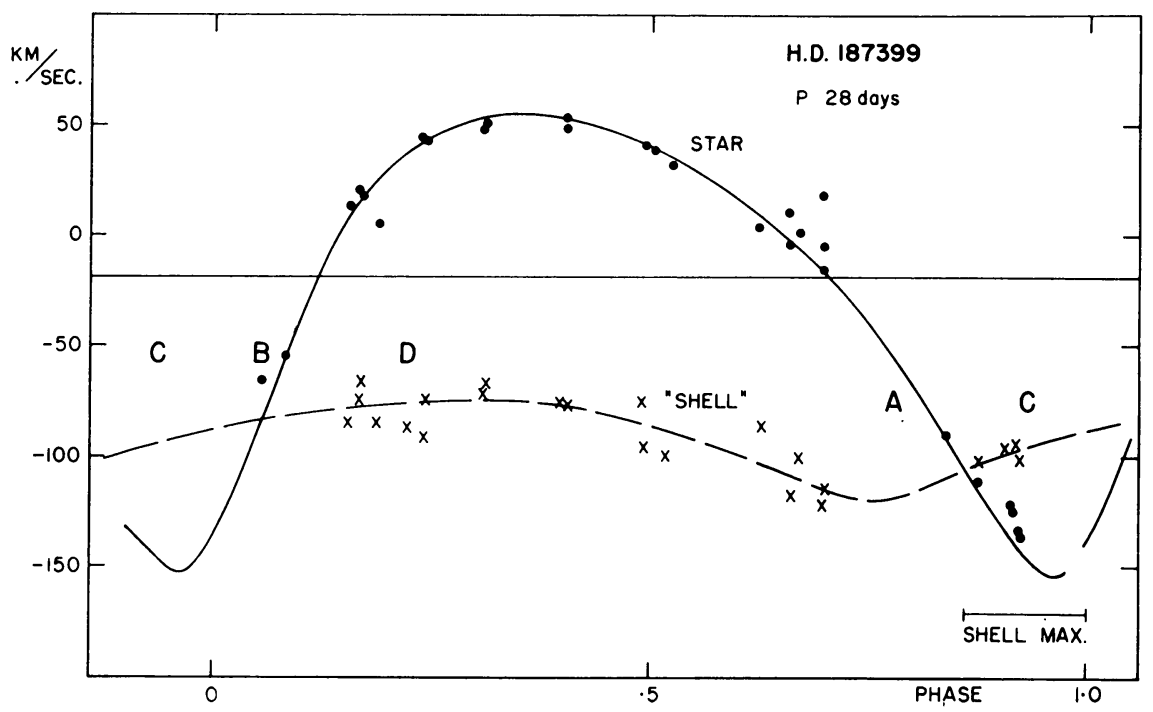

Fig. 3. Radial-velocity curve for HD 187399 from the absorption lines, through the $28 \mathrm{~d}$ period. Dots refer to measures of all lines in the spectrum of the main component; crosses to shell components of $\mathrm{Ca}_{11} \mathrm{~K}$ and Balmer lines.

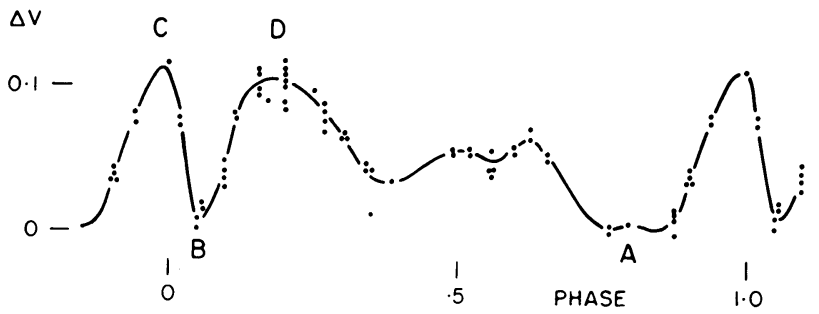

H.D. 187399

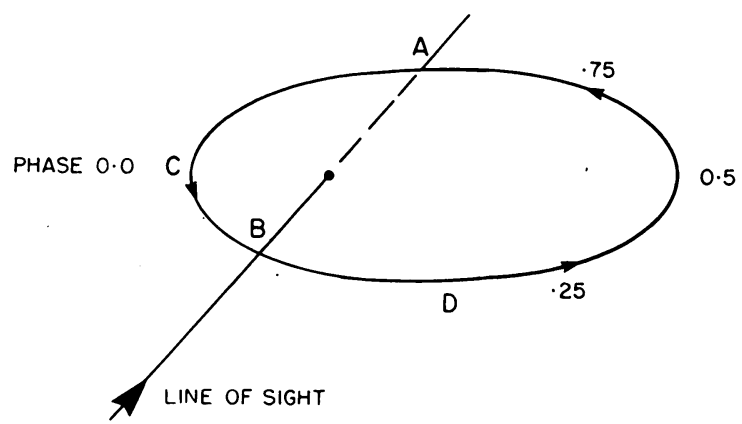

Fig. 4. Upper: Visual light-curve of HD 187399 from two seasons' observations. Lower: sketch of orbital plane, indicating expected phases of eclipse and visibility of gas streams. 
have computed tentative underlying Balmer absorption profiles, which, if correct, suggest equator-on rotation at some $350 \mathrm{~km} \mathrm{~s}^{-1}$.

Figure 4 shows firstly the radial-velocity measurements and derived orbital-velocity curve (the large-amplitude curve) and the velocities of the second absorption system, which has a nearly constant velocity, some $80 \mathrm{~km} \mathrm{~s}^{-1}$ negative of the main curve, but which may show small fluctuations about this with the orbital period, which are displaced in phase. The main curve is derived from $\mathrm{Ti}$ II, Fe II, $\mathrm{He}$ I, N II lines as well as Balmer lines; the lower one shows up only in the Balmer lines and the $\mathrm{Ca}$ II $\mathrm{H}$ and $\mathrm{K}$ lines. The 28-day period has held up for over $20 \mathrm{yrs}$ so that one is inclined to believe the star is a binary. The orbit is eccentric and Figure 4 shows a sketch of the orbital plane. The lower velocity system is modulated so that it is directed towards and away from the observer with extreme values when the component stars are near line-of-sight. One is tempted to think of a gas stream towards the central star and in view of the tentative high value of $e$ to postulate a slow eclipse of it, at phase $\sim 0.8$ and a faster one at phase $\sim 0$. 1 . The third item in the diagram is the light curve (!) as obtained by Graham Hill and Ron Hilditch. These are two season's observations which agree very well, so we are inclined to believe that the variations are real. The points to note are the long low centred on phase $\sim 0$. 8 and the rapid one at $\sim 0$. 1 . Also the high values (phases $\sim 0$. 0 and 0 . 25 ) occur when the postulated gas stream is seen projected against the sky. The amplitude of the effect is $\sim 0 \mathrm{~m} \cdot 1$ which suggests, in conjunction with Figure 2 that the variations are caused by electron-scattering streams.

Figure 5 shows velocity curves for HD 173219. This star has many peculiarities, chief of which are periodic velocity variations which differ in amplitude for different (absorption) lines, in the sense that the amplitude is higher for lines of lower excitation. The diagram shows this effect and the lines are orbital solutions for the lines grouped as shown by excitation potential. The values of $\omega, T_{0}$, and $e$ agree well - only the $K$ 's and (less significantly) the $\gamma$-velocities differ. The eccentricity again is high. There are further interesting data from the (Balmer) emission lines, which change a little in intensity. The absorption lines are not sharp and do not change in strength much. I have no photometry of the star (declination $-7^{\circ}$ is too far south for us) but would like to see some. I would suggest that further work and understanding of these two stars may help us understand the phenomenon of mass-exchange, and have a list of other stars which are probably similar.

Fracastoro: What is the continuous curve drawn through the unfilled circles in Figure 5?

Hutchings: That curve is derived from the unfilled circles.

Scarfe: The effect of the excitation on the velocity curve is similar to that which Ovenden (1963) found in 57 Cyg and explained by the reflection effect. Could this be the explanation here?

Hutchings: There are difficulties. You would expect fantastic changes in the intensities of the lines as they disappear around the other side of the star, and this doesn't happen. I expect we are lookıng at some sort of extended envelope.

Batten: I don't think you can use Ovenden's explanation because no secondary 


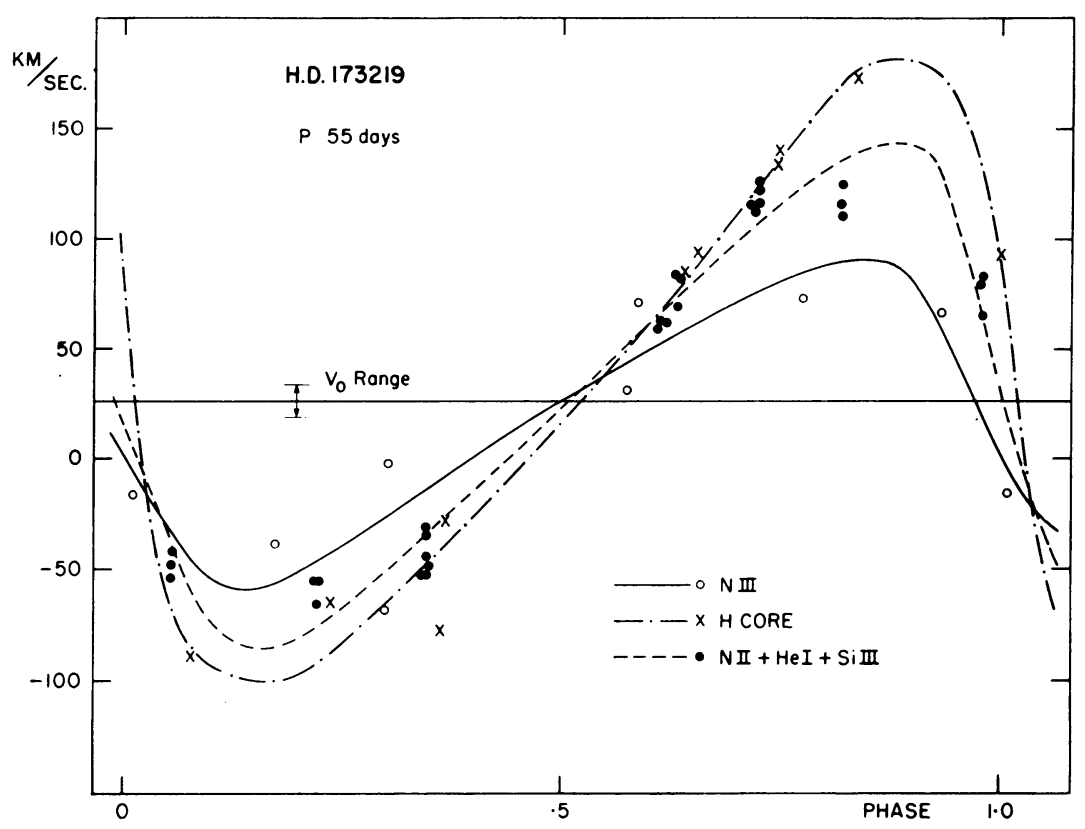

Fig. 5. Radial-velocity curves of HD 173219 from absorption lines of different ions, through the $55 \mathrm{~d}$ period. Curves are orbital solutions based on velocity measurements as shown.

spectrum is seen. You couldn't have the primary star heated up by a secondary not bright enough to show a spectrum - unless that secondary was a very peculiar object.

Hall: I was very interested in your light curves of HD 187399 because a student at Vanderbilt (Tim Frazier) has recently got a somewhat incomplete light curve of the binary DN Cas - a pair of O-type stars. Originally the period was thought to be 1.15 but now we think it's twice that. With the new period, the light curve looks remarkably similar to the one you showed. The only difference is that we have no evidence for unequal durations of the eclipses - as if the orbit of our system is circular. The two eclipses are nearly the same depth. After secondary eclipse the light curve rises quite high, comes down to a plateau, and then the primary eclipse begins. Both eclipses are about $0^{\mathrm{m}} .1$ below the plateau, and the rise is also about $0^{\mathrm{m}} .1$ above the plateau. Since both components of the binary are O-type stars, there might well be an electronscattering envelope. You did say you could get effects as big as $0^{\mathrm{m}} .1$ by electron-scattering?

Hutchings: Yes, that's the sort of change we think could be caused by a hot gas stream.

Hall: Good; I can't think of any other way to explain our light curve of DN Cas.

Devinney: I'd like to mention that C. R. Lynds (1959) observed some B-type stars that showed very strange light curves. Maybe they should be considered in conjunction with what Dr. Hutchings has observed.

Oliver: Do you have the light curve in several colours? That would give some information on electron-scattering. 
Hutchings: Yes, we have the light curve in four colours, and it looks pretty much the same in all four.

Hall: We get the same picture in $U, B$, and $V$ for DN Cas.

F. B. Wood: I think Dr. McNamara's contribution might fit in very well here.

McNamara: Tippets and I have secured observations of the primary eclipse of $U$ Sge over two cycles in the $u v b y \beta$ photometric system. A careful examination of the data indicates that the duration of total eclipse is wavelength dependent in the sense that the duration is greater at longer wavelengths than at shorter ones. This implies either that the B-type star (eclipsed star) is larger at shorter wavelengths than at longer wavelengths or that the opacity of the gas surrounding the G2 IV-III (eclipsing) star is wavelength dependent, being greater at longer wavelengths. The latter possibility seems probably the more likely, and the source of opacity might be the negative hydrogen ion. If this is so, the length of totality should increase for $\lambda>8000 \AA$ since the absorption coefficient of the negative hydrogen ion reaches a maximum at $8000 \AA$.

Hall: You mentioned two possible explanations. In one you assumed the trouble is caused by the cool star, and in the other you assumed it is caused by the hot star. What sort of effect would you predict if there were a slightly extended atmosphere around the hot star?

McNamara: I think that absorption by the negative hydrogen ion is the most likely explanation, because of the behaviour of the $c_{1}$ index during eclipse. If the B-type star being eclipsed was simply a little more extended in the ultraviolet than it was in the yellow, I don't think you would expect the $c_{1}$ index to go down and back up, as it does. So I believe the most likely explanation is in terms of some peculiar absorption effects around the G-type star.

Smak: Are the colour-index values at the bottom of the eclipse of some help to the interpretation?

- McNamara: They don't help out very much, although if you look at the $m_{1}$ index and take it literally as indicating metal abundance, you find that the secondary component of U Sge does turn out to be slightly metal deficient - as I believe is pointed out by Miner (1966). Someone is to discuss the ultraviolet excesses of these stars later. I might say that when you look at the hydrogen lines in these spectra, they seem quite normal," but from the values of the $m_{1}$ index you would have to conclude that the secondary components are slightly metal poor.

$F$. B. Wood: Dr. Smak's comments on radiation from circumstellar matter would fit in here.

Smak: I wish to make a few comments and ask a few questions concerning the radiation of the circumstellar matter. First, there is a great need for quantitative measurements of the emission lines in terms of their absolute intensities. Even from what we know now qualitatively, it appears that there is a large diversity among different types of systems. Yet we do not know whether the absolute intensity of the emission lines coming from disks in the Algol-type systems is systematically greater than in the case of novae and $U$ Gem type systems. Another question is concerned with the visibility of disks in Algol-type systems with primaries of different spectral types. Namely, 
why is it that in nearly all systems with F-type primaries, where emission is present, it is visible throughout the cycle? Is it due to the fainter background of the F-star radiation (as compared with A's and B's), or is it because the emission is indeed stronger?

Passing to the continuous radiation, we may note that it appears to be - relatively much stronger in the case of novae and $U$ Gem type systems, where it often accounts for nearly $100 \%$ of the total radiation of the system. An important observable parameter is then the line-to-continuum ratio. Even in the case of novae and $U$ Gem type systems we find widely different ratios, from cases of rather strong line emission to those where the lines are practically absent. The best eaxmple here is VV Pup, in which the hot spot contributes about $50 \%$ of the total continuum radiation but is practically invisible in the Balmer lines. Before we ask theoreticians to compute meaningful models of disks, spots, etc., we really need more quantitative measurements of radiation coming from these objects.

Underhill: About the real problem of getting absolute intensities, I agree with Dr. Smak. Unless you have absolute energy units, or energy units relative to some standard star, you cannot decide whether the emission is more visible because it is referred to a weaker background, or just because it is stronger itself.

F. B. Wood: Dr. Bolton would like to speak on correlated optical and radio observations of Algol.

Bolton: Algol was first detected as a radio source in the fall of 1971 by Wade and Hjellming (1972). In January of this year Hughes and Woodsworth (1972) and Hjellming et al. (1972) discovered strong radio flares from Algol that sometimes reached intensities in excess of 0.3 flux units. Since the flares were first detected, I have monitored Algol optically to search for spectroscopic changes that might be related to the radio flaring.

The characteristics of the radio flares have been summarized recently by Hjellming (1972). The radio emission from Algol appears to be thermal bremsstrahlung during quiescent periods. During the flare the radio spectral index decreases as though the plasma is becoming partially optically thick. We now have approximately 100 photographic spectra at a dispersion of $12 \AA \mathrm{mm}^{-1}$ that have been taken simultaneously with radio observations at either NRAO or ARO. Most of these show no abnormalities.

However, whenever the radio spectral index is less than about -0.3 we see weak emission in the red wing of the $\mathrm{Ca}$ II $\mathrm{K}$ line. The strength of this emission seems to be correlated with the spectral index in the sense of the emission strength increasing with decreasing spectral index. There is also a suggestion of activity in the $\mathrm{Mg}$ II $\lambda 4481$ line along with that in the $\mathrm{K}$ line.

In view of these possible correlations, I wondered if there might be evidence on on older Algol plates of past radio activity. I was able to inspect over 100 plates of Algol taken by Dr. MacLaughlin with the University of Michigan telescope during the 1927-28 observing season. He usually took three plates per night at a prism dispersion of $17 \AA \mathrm{mm}^{-1}$ at $\mathrm{H} \delta$. About a dozen of his plates show some $\mathrm{K}$-line emission 
and one series shows the emission increasing in strength over about a one-hour period. Thus I'm inclined to think that the radio activity is not a new phenomenon and that it is also not connected with the sudden period changes that are occasionally seen in Algol.

In addition to these possible correlations, we have found that the strengths and line profiles of most of the lines vary throughout the orbit and not just during the partial eclipse of the primary. In particular, we find that the half-widths of the hydrogen lines vary by as much as $75 \%$ and that the residual intensities of the hydrogen lines change by at least $15 \%$ and probably much more. These changes occur in exactly the sense necessary to explain Andrews' photometry of $\mathrm{H} \alpha$ that Dr. Batten discussed. However, the line profiles suggest to me that we are seeing additional absorption outside the eclipses rather than emission during the eclipses.

F. B. Wood: I'm almost afraid to open a discussion on Algol - discovered in 1680 by Montanari, and still there are new findings.

Batten: I'd like to make three comments. First, those results from Hjellming's paper indicate, as I said yesterday, that the radio flare is very probably coming from something other than the stream, if there is a stream in Algol, and it seems to me, therefore, that one should not necessarily expect a close correlation between the radio observations and the kind of emission feature that you have seen at the K-line, which presumably comes from regions where matter is considerably denser. Second, I was very interested in your slide of the hydrogen lines because something much like this happens in the spectrum of $U$ Cep. Most of the time the hydrogen-line profile is basically a very shallow, broad feature. But on a number of plates, which seem to be concentrated around phase 0.5 or $0 .{ }^{P} 6$ after the primary eclipse, you get a definitely deeper profile. The equivalent width is different, the actual width is different, and the depth is different. These two profiles seem to change one into the other quite quickly. I have no explanation for it. On the other hand, there is also definitely at some phases a filling up of either one profile or the other, by something which I think can only be emission from the stream, and it has an irregular profile. So I think there are both types of variation of the hydrogen line present. There is a real change in profile which probably matches what you've shown for Algol, but it isn't quite so dramatic. There is also, I think, a modification of these two basic profiles which is probably emission from the stream. My final comment, which appears in my review paper is that I wish we could find another word than 'flare' to describe this sort of thing. It is a confusing term, and if anyone comes up with another term while we're discussing here, I think we should all agree to use it.

Bolton: I agree that these flares may very well not be associated with the gas streaming although it is hard for me to see how flares of this magnitude could take place without moving some fairly substantial amounts of matter around. I have been intrigued for some time by the so-called B flare stars. There are at least five early-type stars that are reported to show flares of $0.5-0$. 8 . These include a Be star, a $\beta \mathrm{CMa}$ variable, and several ordinary main-sequence stars. As near as I have been able to determine, these are all ordinary stars for their type-except for the reported flares. 
I have looked at three of these stars and have good evidence that all three are binaries, and I wonder if these are related in some way to what is going on in Algol. Finally, I think that it should be emphasized that the correlation I have found is very tentative. We have additional simultaneous observations scheduled for the next month so that we may perhaps firm up our conclusions.

Van't Veer: If you wish to correlate a flare, or let us say outburst, with a period change, you have to wait a very long time. With a period change of one second, you have to wait for the accumulation of many seconds before you can see it - one or two years.

Bolton: What I am saying is that if the correlation between the $\mathrm{K}$-line emission and the radio flaring is real, then the emission on the 1927 plates indicates that the radio activity is not correlated with a period change because there was no period change in 1927.

Plavec: How does an outburst in Algol compare with a solar outburst?

Bolton: The radio outburst is much stronger. If Hjellming is right about Algol being a transient $\mathrm{X}$-ray source near the outburst peak, the energy output would be $\sim 10^{35}$ ergs s$^{-1}$.

Plavec: The variation in hydrogen-line profiles both in depth and width is observed not only in the spectra of Algol and U Cep, but also, for example, in those of RW Tau and $U$ Sge. So one is tempted to regard it as typical for primary components of Algol systems. But all these stars are of spectral types very close to B8 or B9, and it might be interesting to see if this variation does not occur in single, normal stars of type B8 or B9.

Hill: Somebody had seen K-line emission on plates of Algol taken a number of years ago at the Dominion Astrophysical Observatory, but nothing was ever published. I'd also like to comment that one must be careful in interpreting the spectrum of Algol in primary eclipse, because the line profiles can be distorted by the spectrum of the third component.

Popper: What is the size of the radio error-box from which this variation occurs?

Bolton: Four-tenths of a second of arc.

Whelan: Are there any polarization observations?

Bolton: No, not yet. We hope some may be made on the next run.

Smak: I have a question for Dr. Popper. At first your question seemed quite funny, but did you mean to imply that you suspect the third body to be responsible for the radiation - or any other field star located nearby?

Popper: I just wanted to inquire if there was any possibility of a coincidence. It's quite unlikely, I suspect, but we should ask if the radio emission really does come from Algol.

Smak: Could the third body be responsible?

Bolton: I think the radio errors are such that that would be permissible. I think that the argument that has been made against the third body being involved is that in the one case (Antares) where the radio emission in a binary system can clearly be assigned to one of the component stars the emission was from the B-type star and not the 
companion, which was losing mass. This is perhaps not too strong an argument, but it was what led Hjellming to look at other B-type binaries where there was mass exchange. There is the additional argument that the size of the plasma cloud involved in the flaring seems to be about the size of the Roche lobes about the close pair in the Algol system.

Fracastoro: I wouldn't dare to ask you to go on observing this radio emission in order to see whether there is any correlation between the radio-outburst frequency and the orbital period of the third body, since you have already found that there is no correlation with the 2.86 photometric period. It would be very interesting to find something like the 11-yr solar cycle.

F. B. Wood: We are deliberately keeping time for discussion. Anyone who has signified a wish to speak will get an opportunity. We are altering the order of speakers a little. A group on period changes should be kept together at some session. Dr. Walter will now talk to us on Magnetic Fields and Hot Spots.

Walter: I mentioned this problem yesterday, in connection with SW Cyg. A young colleague and I have a paper on TW And in press (Ammann and Walter, 1972). This system has period of four days, and we believe that the existence of hot spots on the surface of its primary component can be clearly demonstrated.

The light curve of this system seemed to be without irregularities, but because of the possibility of gas streams within the systems we tried to base the rectification only on the second quarter of the light curve, which, in many systems, seems to be free of the effects of gas streams. We call this method 'short-region rectification' in contrast to the usual 'long-region rectification' in which the whole light curve outside eclipses is used. The short-region rectification showed the presence of additional light before and after primary eclipse. We interpreted this as the light of hot spots. The interpretation seems to be supported by the observed intensities in $B$ and $V$ during the primary eclipse when the additional light is also totally eclipsed - very similarly to the situation in SW Cyg. Again, in TW And, the hot spots seem to be located at high latitudes and thus hint of the existence of magnetic fields. The short-region rectification not only gave a better representation of the normal points outside eclipses than the usual rectification, but the sum of the squares of the residuals of the intensities within the primary eclipse was also much reduced by the new method of solution.

In Algol systems, we have to distinguish between stable geometrical elements and variable properties which arise from gas streams and/or magnetic fields. This could be shown, for example, by a comparison of Dugan's photometric observations of RV Oph in 1906-14, with my own obtained in 1964. Another system which may show variations of the light curve is TV Cas $(P=1.8)$ In 1971 its light curve was of the kind to be expected from systems containing gas streams. For such systems it may be advisable to wait some time until the system exhibits a light curve that can be explained by some known model. Catalano's (1966) rectification of the light curve of u Her provides an example of the great differences which may result from different methods of rectification. Short-region rectification of this light curve gives results that strongly suggest hot-spot activity and UV-radiation from the spots. 
Smak: Where does the evidence of magnetic fields actually come from?

Walter: From discussion of the observations of the systems SW Cyg and TW And. The geometry of the eclipses is well known, and it can be shown that the luminous regions are situated on the surface of the primary component at about latitudes $\pm 60^{\circ}$ out of the orbital plane. The additional light of the hot spot is seen outside eclipse for about one half the orbital period. It is fully seen at the beginning and the end of the primary eclipse. Within this stellar eclipse there is a second one - a total eclipse of this additional light - and it is possible to determine the region in which this light must be situated as is shown in my paper on SW Cyg (Walter, 1971).

Smak: I have two comments. First, the lines you drew actually correspond to the beginning and end of the eclipse of the spot. If the spot is slightly elongated, it can be on the equator and show the same effect. Second, all the numbers that enter, namely the radii, inclination, etc. are based on your specific solution. You use only parts of the light curve, and this determines the answers. Since you admit that there is a hot spot, or detached material, it becomes a matter of judgment or arbitrary decision which part of the light curve you use.

Walter: We have made a photometric solution of a rather deep total-eclipse curve, and the distortions of the light curve, which have been influenced by the method of rectification, concern only a few per cent of the intensities. The photometric elements cannot be changed very much and still give a reasonable solution. I think we have indeed obtained a geometrical solution that is close to the truth.

Kitamura: Do you think that the existence of a magnetic field provides a unique solution for the residuals?

Walter: It seemed to me the simplest hypothesis and I did not try any others.

Kitamura: Your solution for the light curve is strongly based on 'short-region rectification'. I think that any theory of rectification is approximate. In order to treat such a peculiar light curve you must check the rectified light curve in various ways. In particular, did you test whether your rectified light curve, outside eclipse, can be represented well by the eclipse function?

Hall: To put the same question another way: if you turn the problem around and solve the primary eclipse, would that solution represent the light curve out of eclipse?

Walter: I see. First, we tried to rectify the whole light curve outside eclipse. The comparison of the result with the observed curve was not satisfactory. We could obtain a satisfactory comparison from the short-region rectification, but once we had made this, it was difficult to return to the old method. The original light curve seemed quite symmetric, normal, and free from the effects of gas streams.

Kitamura: Even so, in my opinion, with such a peculiar system we should be very careful with rectification and we should check that the light curve within eclipse can be well represented by the eclipse function only.

Milone: You mentioned the need to look at these light curves over many years, and I would like to emphasize this very strongly. O'Connell (1951) published a list of systems whose light curves show unequal maxima. We have observed a few of these 
again, and found that the sign of the difference between maxima has changed. A couple of these cases have been very well studied, RS CVn by Catalano and Rodonò (1967) and RT Lac by Doug Hall and myself. We do in fact find some sort of longterm cyclic variation and we try to take it out of the light curve before we make a solution. It seems to me to make sense to look at long-term changes in the light curves as well as to examine those parts of the light curves that show short-term changes. We can remove the time-dependent part of the light curve and try to rectify in the usual manner.

Walter: Yes, we considered variations that might have happened during the years of observation. There were some small variations just before and after primary eclipse, but they were so small that we could neglect them.

Linnell: It's surprising that you were able to obtain acceptable values of rectification coefficients with such a short phase range. I think that Dr. Kitamura's point is very pertinent. I wonder whether the nature of the residuals would be different if you had chosen short phase-range segments in different phase regions, rather than just on either side of the secondary minimum. In other words, how sensitive is the nature of the residuals to the particular phase range chosen for the determination of the rectification coefficients?

Walter: I do not have the exact figures here, but, of course, for both rectifications we used the method of least squares. The coefficients $A_{1}$ and $A_{2}$ of the $\cos \theta$ and $\sin \theta$ terms ( $\theta$ is the phase angle) had very small mean errors as determined from the long region. The errors of these terms in the short-region rectification are, of course, larger, but the values of $A_{1}$ and $A_{2}$ are significant and may be used for rectification. I don't believe our solution is exceptional. I think TW And is a quite normal Algol-type system.

Biermann: I think your geometric argument is based on the assumption that you see the same part of the hot spot as it goes into and comes out of eclipse. As Smak said, it might be an elongated region of which you see different parts at the two phases, and then you can't use your argument. Even assuming that your picture is correct, magnetic fields would have to be rather high. Have you looked for any evidence of that in the spectrum?

Walter: I have no instrument for it, but I think that fields of $100 \mathrm{G}$ would suffice. In your own dissertation, you mentioned that fields of this order of magnitude would follow from estimates of the kinetic energy and magnetic energy of particles. Magnetic fields of this order of magnitude could affect the gas streams but could not be detected from the spectra.

Hilditch: You mentioned light curves of $\mathrm{u}$ Her. There is a red curve by Catalano and a blue one by Ruiz (Merrill, 1963). I have made a preliminary analysis of these light curves, using the synthesis programme of Graham Hill and John Hutchings, and I can fit the light curves very well with the normal model. All I need to fit the light curves completely are improved values of the mass ratio and of the temperature of the primary component. It is not necessary to introduce any gas streams.

Walter: It is still unexplained, however, that the long-region rectification of the 
excellent light curves by Shapley and Calder (1935) and Catalano are not satisfactory at all.

Cowley: What are the spectral types of the components of TW And and of the other systems in which you find hot spots?

Walter: The components of TW And are F0 V and G6 IV. The brighter component of SW Cyg is an A-type star.

Plavec: What are the radii of the two stars, for example in TW And?

Walter: One is about 0.16 and the other 0.25 of the distance between the centres of the stars. The larger star fills its Roche lobe.

Plavec: We should realize that normally the subgiant is relatively large in comparison with the other star. Therefore, the nozzle (or whatever it is) through which the material flows from the late-type star is not very small - maybe larger than, or of the same size as, the radius of the other star. We are discussing whether the hot spot is very near the equator or near the pole. In either case we are assuming that the stream is very nicely focussed from this relatively large nozzle into a relatively small spot where it hits the primary star. I can't say this assumption is wrong, but we should bear in mind that it requires very strong focussing of the flowing material.

Hutchings: I'd like to refer back to Dr. Hilditch's remarks, and make a comment on behalf of those in the room - and there are several - who don't believe in rectification. It seems to me that rectification has been controversial, even among those who do believe in it. I would like to see this sort of system analyzed by the synthesis method. If we can produce the same sort of residuals by a totally different approach, we might well be much farther along the way to establishing the existence or otherwise, and the positions of these hot spots. It seems that we are talking about magnetic fields and such like things a little bit prematurely.

Van't Veer: I agree with Hutchings that we can do better now with the method of synthetic light curves. You should try to reproduce the light curve artificially and compare your synthetic light curve with your observations. It is a method of trial and error, but it avoids the use of unreasonable physical hypotheses.

Walter: You can use the method of artificial light curves if there are no gas streams present. If there are no gas streams, the short-region rectification should give the same result as the long-region rectification - but, in fact the two give different results.

Smak: I am one of those who has never done any rectification, but it is my understanding that rectification, in the meaning of the conventional Russell method, is also making use of a systhetic light curve based on very specific assumptions; namely, there are two stars, one or both of them being possibly elliptical and possibly affected by a reflection effect that can be described by a cosine term. If we have something else in the system, either from the very beginning, or as a result of our rectification, then the Russell rectification procedure should not be applied blindly.

F. B. Wood: Blindly! Blindly, no. I'm tempted to say more, but I'd better not! We spent three days on this subject in Philadelphia, a year ago. I will just say that we are moving into an area in which there is intense disagreement amongst those working in it. 
Oliver: If you do believe that there might be spots, either hot spots or dark spots, on a star, neither traditional rectification nor synthetic light curves are really going to show you them, because they are not included in either of those models. Either way, all you will see is a slightly greater scatter - or, if you are lucky, some systematic trends - in the residuals. If you are not willing to accept the possibility of some other model and try it, for example by not fitting your synthetic light curve to points in the region where you suspect some anomaly, you are not going to find the anomaly. Even in the synthetic-light-curve method, you should at least try 'short-region rectification' by leaving out the region on either side of primary minimum when fitting your synthetic light curve and then looking to see if the same trend is found in the residuals. You cannot judge, I think, whether or not a hot spot is present from the general scatter of the residuals. Who's to say how much scatter there should be? The scatters in the two plots we saw did not differ by more than a factor of two. Who can judge what the real scatter should be, a priori?

Chen: I agree with John Oliver's argument and I think that at the IAU Colloquium No. 16 in Philadelphia we showed, at least the invited speakers showed, that the solutions for an Algol-type light curve, using the synthetic-light-curve technique and the rectification technique separately were the same. The differences of elements were in the third or fourth decimal. This, I think, is beyond the accuracy of the observations.

R. E. Wilson: I don't believe we should complain about someone failing to use a light-curve-synthesis program when he does not have access to one. Such programs do not exist everywhere in the world. I would like, however, to comment on the free-hand light curve Dr. Walter drew through his normal points of TW And. I think it is not a good practice to publish hand-drawn curves through the observations. One can publish only the observational points and see what the observations are like, or one can publish the observations with a theoretical curve through them, which then shows the comparison of theory and observation. But a hand-drawn curve through the observational points only misleads the eye, and, as far as I can see, serves no purpose whatsoever, although many people publish them. I would like to see this practice ended.

Walter: I can answer you directly: this curve will not be published! We are publishing only the normal points and the observations. Nothing has been deduced from the curve - it's only to help your imagination.

R. E. Wilson: My point is that it hampers the imagination. With a hand-drawn curve through the points, it is difficult to judge the run of the observations.

Walter: Then, please, think away the line, and look only at the points! The line should only show that the light curve, which was composed of some hundred observations from about eighty nights, is smooth. The observations don't show much scatter. The line was not meant to make one think of hidden problems of gas streams.

Linnell: It seems to me that in general the attempt to detect photometric anomalies in systems such as this where you believe that there may be gas streams present, has to depend on residuals from an accurately calculated light curve whether you are going to do it by synthesis technique or whatever. The detection of trends which are 
statistically significant must depend on extensive observational residuals. I think that we should try to get away from the use of normal points. In view of the discussion yesterday concerning the transient nature of some of these phenomena, the assembly of individual points into normal points from several different cycles may, in fact, tend to hide some of the photometric effects that you hope to investigate. When you construct a synthetic light curve, and then calculate residuals, it's very important to look at the individual residuals on each night, and compare those residuals with those of the extinction star from the extinction line, so that you can determine whether or not there is genuine statistical significance to postulated trends in the residuals of the variable.

Walter: For just this reason, my method was to avoid giving single nights too much weight or preference. I make only three or four observations a night in each colour - especially outside eclipse - and combine the results of many nights. I think, when this method is used, it makes no difference whether we use normal points or single observations. Normal points can be helpful, and often help us to see more from the observations. I doubt if we would have arrived at what we think is a solid interpretation of an interesting property of TW And if we had used only single observations. We would have given all the observations to a computer and would have taken the results and published them. We would have no need to consider further, because we would have considered the problem solved.

F. B. Wood: We are well over schedule, but the participants have been interested. Let's have one last comment from Dr. Leung, and then we must continue either privately or at a later session. This communication illustrates well that any good light curve creates a lot more problems than it solves!

Leung: A very good example of a variable light curve is provided by the W UMa system VW Cep. If you realize that some phenomena will not be very stable so that there are likely to be cycle-to-cycle variations, you can always pick a season of a few weeks' time and solve the light curve from a few particular cycles. You can account for all sorts of distortions by hot spots, cold spots, or gas streams and then obtain the orbital elements. This will not be very meaningful bacause the normal points from the next few months will define a light curve that will give you a completely different set of geometrical elements. It's rather dangerous to introduce too many complications into the solution of a light curve obtained over only a short period of time. I don't use normal points from, say, two weeks, but I try to use all the individual observations to define a mean light curve and look for periodical fluctuations from it. In this way, I try to separate whatever is periodic, transient, or non-secular. Then I have a light curve I can analyze.

\section{References}

Amman, M. and Walter, K.: 1972, Astron. Astrophys. (in press).

Baglow, R. L.: 1948, Monthly Notices Roy. Astron. Soc. 108, 343.

Batten, A. H.: 1968, Publ. Dominion Astrophys. Obs. 13, 119.

Blanco, C. and Catalano, S.: 1972, unpublished.

Catalano, S.: 1966, Publ. Catania Oss., No. 97. 
Catalano, S. and Rodonò, M.: 1967, Mem. Soc. Astron. Ital. 38, 395.

Fletcher, E. S.: 1964, Astron. J. 69, 357.

Hall, D. S.: 1972, Proc. IAU Colloquium No. 16 (in press).

Hjellming, R. M.: 1972, Nature Phys. Sci. 238, 52.

Hjellming, R. M., Wade, C. M., and Webster, E.: 1972, Nature Phys. Sci. 236, 43.

Hughes, V. A. and Woodsworth, A.: 1972, Nature Phys. Sci. 236, 42.

Huffer, C. M. and Kopal, Z.: 1951, Astrophys. J. 114, 297.

Koch, R. H.: 1960, Astron. J. 65, 326.

Koch, R. H.: 1962, Astron. J. 67, 130.

Kron, G. E.: 1947, Publ. Astron. Soc. Pacific 59, 261.

Lynds, C. R.: 1959, Astron. J. 130, 577.

Merrill, J. E.: 1963, in F. B. Wood (ed.), Photoelectric Astronomy for Amateurs, MacMillan, New York, p. 165.

Miner, E. D.: 1966, Astrophys. J. 144, 1101.

O’Connell, D. J. K.: 1951, Publ. Riverview College Obs. 2, 85.

Ovenden, M. W.: 1963, Monthly Notices Roy. Astron. Soc. 126, 77.

Rodonò, M.: 1967, Mem. Soc. Astron. Ital. 38, 465.

Sanford, R. F.: 1951, Astrophys. J. 113, 299.

Shapley, H. and Calder, W. A.: 1935, Harvard Circ. No. 398.

Wade, C. M. and Hjellming, R. M.: 1972, Nature 235, 270.

Walter, K.: 1971, Astron. Astrophys. 13, 249. 